\title{
Epistemology of Material Properties
}

\author{
Joachim Schummer \\ Dept. of Philosophy, University of Karlsruhe, D-76128 Karlsruhe, Germany \\ Joachim.Schummer@geist-soz.uni-karlsruhe.de
}

\begin{abstract}
This paper presents an epistemological approach to the investigation of material properties that is opposed both to phenomenalistic epistemology and recent linguistical and ontological accounts of matter/mass terms. Emphasis is laid on the inherent context dependence of material properties. It is shown that, if this is taken seriously, some deep epistemological problems arise, like unavoidable uncertainty, incompleteness, inductivity, nonderivableness. It is further argued that some widely held epistemological accounts, namely that of essentialism, constructivism, and pragmatism, all reveal some serious defects if related to the recognition of materials. In order to responsibly manage our material environment, a more realistic estimation of our epistemic abilities and prospects is suggested.
\end{abstract}

Keywords: epistemology, material properties, context dependence, experiment, chemistry.

\section{Introduction: The primacy of epistemology}

Since a couple of years we may witness a growing philosophical interest in matter(s), material substances, or material beings [1]. Far from their Aristotelean-Thomistic ancestors, today's philosophers focus on the ontology of matter(s) or the linguistics of mass terms by logical means, provided by analytical philosophy mainly in a Quinian manner. While this has enabled considerable insight in logical and linguistical features, it suffers from a certain sterility with regard to ordinary and, in particular, scientific experience of material/chemical substances. In fact, most accounts leave epistemological problems and presuppositions undiscussed or implicit.

Against a (still) prevailing tendency to tell ontological stories based on logical-linguistic analysis, I claim for the primacy of epistemol- 
ogy, i.e. an analysis of the conditions, possibilities and limits of our knowledge gaining processes, that may lead, only in a second step, toward a (linguistically shaped) world view. There is no doubt that linguistic conventions are important constraints of, and even influential on, the social process of knowledge gaining, esp. in science. But these constraints only play a conservative role, while our knowledge of materials tends to change and, as I will show, improve by experimental and conceptual refinements. Moreover, ignoring the non-linguistic constraints, in particular the epistemic conditions of experiencing materials, is even in danger of missing the meaning of our scientific (mass) terms.

Starting from an 'epistemologized' ontology of matter, I will point out some deeper epistemological problems of material properties, that arise mainly from its inherent context dependence. It turns out, that these problems forces us towards a more modest epistemological position: neither skepticism, nor naive optimism.

\section{Ontology epistemologized: material objects}

The problem of telling a 'pure' epistemological story is that it seems to presuppose an ontological starting point: "What is matter?", or the be more precise: "What are material objects?". Since Aristotle's famous analogical reasoning (Physica, 191a 8) there was a tendency to refer to some intuitive pre-understanding. So called 'materialists', in saying that 'everything' is matter, did and do refer to such a putative common intuition, while actually missing the point of clarification. On the other hand, epistemologists of the modern era essentially depended on ontological commitments of mechanistic philosophy: to be a material object was meant to have spatial extension (Descartes), to be localized by spatial coordinates (Newton), to bear primary qualities (Locke) or to be 'formed' by the Anschauungsform space (Kant). Not only did these arbitrary metaphysical (or transcendental) assumptions break with a 'pure' epistemological approach, their strange emphasis on spatial attributes even hindered a real epistemology of material investigation. For, though the philosophical dream is still alive, material science is not (and was never) a subdiscipline of geometry [2].

The starting point of our epistemologized ontology is simply this: to be a material object means to be capable of being an object of material investigation. This definition is neither subject to the above criticism nor imprecise, as it turns out, when we clarify material investigations (s.b.). Thus, 'material' is a dispositional predicate like any other material predi- 
cate (s.b.), and as such, its correct application can follow operational rules: whenever we are uncertain, if $x$ is material, then we should check if $x$ is capable of being an object of material investigation.

Note that $x$ might also be an object of different kinds of investigation/experience, e.g. physical, morphological, economical, astrological, aesthetical etc., according to different perspectives on the object. Our epistemological approach does not intend to bring about a metaontology. But before we discuss material investigations in detail, we may stress some general traits of the material perspective. The material perspective abstracts from all extensive and spatial properties such as coordinates, size, (micro-)structure, or absolute mass and in some respect even number, just as it abstracts from personal and economical value, magical meaning etc. That does not mean at all that material objects are macroscopic and continuous bodies, as a widely held but misleading metaphysical doctrine of matter claims. Instead, looking upon an object from the material view point means: I do not care about its seize (mi$\mathrm{cro} / \mathrm{macro}$ ) and structure (continuous/discontinuous); I have no interest in its global spatial coordinates, and I even ignore prima facie, whether the object may consists of two, three or a thousand drops, crystals or any mechanico-geometrical parts.

\section{Against Phenomenalism}

According to Locke's contemporaries, material properties like colors were secondary qualities, essentially dependent on our sensory constitution and caused in a way by some underlying primary qualities which were the 'real' material properties. The later phenomenalistic and positivistic tradition skipped the metaphysical account of 'real' properties in order to take phenomena or qualia as the one and only evident data basis for any empirical knowledge. While logical positivists tried to reconstruct the empirical basis in linguistic terms ("here, now: red"), modern naturalist try to explain them again in physiological terms ('neuron activation'). That is, in short and very roughly speaking, the philosophical story of the epistemology of material properties: a pursuit for basic, elementary, and context independent constituents of material knowledge.

The scientific, i.e. chemical, story, on the other hand, goes just the opposite way. Material predicates were always attributed to something out there, something that was manually made an object of experimental investigation before, a material sample. Sensations were, for sure, always important in experimental science, but scientists overcome the 
naive correspondence between, say, a red sensation and a red object. The (red sensation based) sentence " $x$ is red" actually contains no empirical information about $x$ at all, because everything looks red under red light, i.e. 'red' is no material predicate. The striking difference between phenomenalists and scientists is that the latter make the contextual conditions of experience as explicit as possible, i.e. scientists use "red under conditions $c_{1}, c_{2}, c_{3} \ldots$ ". as a material predicate. Consequently, "red under conditions $c_{1}{ }^{\prime}, c_{2}, c_{3}{ }^{\prime} . .$. ” is quite a different material predicate, and so on.

Phenomenalism is an epistemology of common sense, of ordinary experience when we implicitly presuppose our usual standard contexts of daily life. Material science, on the other hand, aims at understanding by questioning our self-evident and implicit assumptions, and it does so by varying contextual conditions to the very extremes. Understanding means first of all: building concepts as precisely as possible to distinguish material objects in an unambiguous way, in order to build a classification of materials. Epistemologists and philosophers of science have stressed too much the role of truth, while neglecting the problems of building and refining empirical concepts. A sentence like " $x$ is red" is, strictly speaking, neither true nor false, because it contains no empirical information about $x$ (s.a.). And there is no serious way at all (pace Quine) to define truth conditions more precisely by referring to stimuli of our nervous system. That is completely the wrong track.

\section{Material properties according to contextual conditions}

Our task is now to clarify (scientific) material investigations, i.e. the various ways to determine material properties. A material property is reproducible behavior within certain reproducible contextual conditions. The contextual conditions in question can be made explicit by distinguishing contextual factors: (1) mechanical forces, (2) thermodynamical factors (temperature, hydrostatical pressure), (3) electromagnetic fields, (4) other material objects (chemical substances), (5) biological organisms, (6) ecological systems. Every context of investigation can be specified with respect to each of these factors. The crucial step to systematize material properties is the definition of standardized factors for each case (excluding external forces, shielding electromagnetic fields, controlling thermodynamical standard conditions, working with abiotic and closed containers of inert materials). Now, we can turn our attention to selective factors of interest and vary them in a controlled manner while the others are kept standardized. 
The striking point is, that scientists distinguish material properties according to the interesting contextual factor. Thus, we have: (1') mechanical properties (like elasticity), (2') thermodynamical properties (like specific heat capacity, melting point), (3') electromagnetic properties (like specific magnetic susceptibility, specific electric conductivity, optical absorption coefficient), (4') chemical properties (like the capacity for oxidation or the solubility in a certain liquid), (5') biological or biochemical properties (like $\mathrm{LD}_{50}$, antibiotic or anesthetic effect), (6') ecological properties (like ozone depletion potential, greenhouse effect factor). If two or more interesting factors are combined, we get 'mixed' material properties (photo-chemical, thermo-electrical, thermo-electrochemical etc.) [3].

On the other hand, we can also give a corresponding list of types of behavior: change of mechanic form, electromagnetic state, thermodynamic state, chemical identity, biological and ecological state. But it is actually impossible to systematize material properties according to different types of behavior. For, first, we usually have some combination of behavior. And, secondly, the non-behavior in certain contexts, e.g. the non-reactivity with certain reagents, non-toxicity for certain organisms etc., are important material properties as well.

\section{Epistemological problems of material properties}

There are two striking features of material properties which both raise a lot of epistemological problems: context dependence and change. Change is of central importance esp. in chemistry, because determining a chemical reaction property means changing the object's chemical identity [4]. In chemistry the metaphor of the uninvolved observer, that today puzzles so many philosophers of quantum mechanics, had never a right. In what follows, I will concentrate on problems that arise when we take context dependence of material properties seriously.

\subsection{Intricate relationality}

Our favorite linguistic subject-predicate scheme is challenged when we try to attribute material properties. Why shall we attribute the behavior of our experimental system only to one material object instead of all components? The problem is most obvious if we address chemical properties, where we have at least two material objects whose common behavior is under investigation; and after mixing them they even cannot be distinguished any longer. Moreover, if any chemical reaction occurs, 
which means a change of chemical identity, our former objects vanish. To what object then shall we attribute the chemical reaction behavior? In fact, material properties, esp. chemical properties, are intricate relations. The simplest case of two material substances $a$ and $b$ reacting to $c$ and $d$ is at least a four-fold relation $(a, b) R(c, d)$, with two separate sets of variables. Only if $b, c$, and $d$ are chemically well defined specimens of material types $B, C$ and $D$, and if $a$ is our material object under investigation, then we can attribute to $a$ the chemical predicate: 'being reactive with material type $B$ to types $C$ and $D$ '. Correspondingly, we can attribute a second type of chemical predicate to $c$ : 'being a possible co-product of material type $D$ from reaction of type $A$ and $B$ '.

\subsection{Nonderivableness}

Any precise definition of a material predicate already requires precise definition of material predicates for unambiguously describing the corresponding material context. Material predicates cannot be founded on some basic set of context-independent predicates, because there are no such, e.g. in material science the dream of logical positivism was definitely on the wrong track.

Alternatively, a given set of vague material predicates, implicitly bound to ordinary contexts, must be stated more precisely and enlarged in many dialectical steps: Determining contexts more precisely provides more precise predicates to determine in turn contexts more precisely, and so on. Thus, in material science we have some kind of dialectical improvement of knowledge of materials, instead of logical combinatorics. And that is exactly why our list of contextual factors (sect. 3) was so full of assumptions based on present knowledge.

\subsection{Inductivity}

The difference between dispositional and non-dispositional (manifest) material predicates is not based on context dependence, but on the pragmatical difference between intended instances of attribution. By saying " $x$ melts under contextual conditions $y$ " I can either (a) describe the singular behavior of $x$ in a certain context $y$ or (b) express my expectation that $x$ will always melt whenever it is exposed to contextual conditions of kind $y$. The so called theoretical or law-like character of dispositional predicates does not arise from their nonderivableness from fictional basic predicates (as Carnap put it), but from the inductive usage which shifts from (a) to (b). If an empirical statement $F(x)$ means that we can experience $F$ with $x$, whenever we want, then there is no empirical 
predicate without inductive use. But that does not mean, that $F$ is not empirical, otherwise there would be no empirical predicate and no empirical science at all. $F$ is empirical, just because the intended instances of attributing $F$ are empirical contexts.

\subsection{Uncertainty}

Material predicates bear an unavoidable element of uncertainty. If the precise definition of a material predicate requires the precise description of the context, then we need, strictly speaking, a complete description of the actual state of the world. The obvious cognitive problems are joined by logical and linguistic problems. Dispositional predicates must be applicable to infinitely many situations of the same material object (induction from (a) to (b)), although the state of our world is changing. It must also be useful in many other instances for many other people, otherwise it is practically worthless. That problem can be tackled only by looking for relevant contextual conditions on which the behavior of the material test object significantly depends, i.e. by looking for functional laws. Since there is no guarantee for the completeness of relevant conditions, we will always have an residue uncertainty.

\subsection{Incompleteness}

Material predicates are created by inventing new experimental contexts. There seems to be no limit to the possibility of new experimental contexts, except for the limits of our imaginations. Thus, the number of material properties suitable for characterizing a particular material object can be increased ad libitum. This is again of special importance for chemical predicates because of its special relational structure. To create a new chemical context means to choose a new combination of reaction partners and conditions. The immense proliferation of new chemical substances (only 1996 chemists produced 1.3 mio new ones)[5] goes along with an exponential proliferation of new possible chemical properties. As a consequence, our knowledge about material properties will always remain incomplete [6].

\section{Abandoning epistemological myths}

Realism, taken in the ordinary language sense of realistically estimating our epistemic prospects, forces us once more to argue against epistemic optimism with regard to knowledge of material substances. In particular, there are three epistemological myths to abandon in chemistry [7]. 


\subsection{Myth of essentialism}

The essentialistic myth (a heritage of the doctrine of primary qualities) tells us, that the essence of every material substance is hidden in its inner structure. (A recent semantic variant of this tale (H. Putnam [8]) seriously claims that a certain structure is even the proper meaning of a mass term.) According to this myth, in order to achieve complete knowledge about a substance, we need only depict a three-dimensional structure very exactly. Such a picture would wondrously bear all the information about our awfully context-dependent material properties. Just look at the molecular structure of chloroflourocarbons and you will see its ozone depleting potential. Regard a picture of thalidomide and you see that it causes malformations in newborns when taken by pregnants. However, the myth keeps quiet about the fact that reading the picture dynamically requires first of all a translation into quantum mechanical terms. Moreover, it implicitly presupposes quantum mechanical models of our atmosphere, of pregnants, of every possible empirical context, i.e. of all possible material worlds.

\subsection{Myth of constructivism}

With the attitude of enlightened criticism against essentialism the constructivistic myth tells us, that 'real' knowledge of an object lies in the conditions and methods of its construction. According to the verum factum principle knowledge is proved by our productive abilities. Friedrich Engels, for instance, explicitly argued this way when regarding chemical knowledge [9]. But this tale simply ignores the fact that producing material substances does not require a lot of knowledge. In fact, producibility is just a single chemical property (s.a.) among infinitely many others. And chemical properties are just a single type of material properties among many other types. We should be aware that environmental problems arise just because we know too little about the thousands of new substances, that we produce so easily every day.

\subsection{Myth of pragmatism}

According to the myth of pragmatism, knowledge about an object should be searched in its practical use. If we are able to utilize an object for our own purposes, then we definitely know everything a human being can know about it. Variants of this tale may be found in every variety of pragmatism, recently modified by Hacking in arguing for the reality of utilized theoretical entities [10]. Interestingly enough, it was again 
Engels who explicitly uses this tale with regard to chemical substances by referring to the economical success of his contemporaneous chemical industry (ibid.).

Here we meet again the same fallacy of taking only a few properties for the whole story. That is exactly how we look upon materials in daily life, by hypostasis of our favorite functional properties: some materials are good for eating ('food'), some are good for coloring ('colors'), others are good for clothing ('cloth') etc. But our material world is much more complex than this tale and the others want us to have it.

Epistemology of material properties leads us to a more modest estimation of our epistemic prospects. Our findings of uncertainty, incompleteness etc. are certainly less spectacular than those of quantum mechanics or mathematics. But I am afraid, that they are getting more and more important in managing our future material environment.

\section{References}

[1] E.g. F.J. Pelletier (ed.), Mass Terms, Dordrecht: Reidel 1979; F.J. Pelletier, L.K. Schubert: "Mass Expressions", in: Handbook of Philosophical Logic, vol. 4, Dordrecht: Reidel 1989, pp. 327-407; P. van Invagen, Material Beings, Ithaca: Cornell Univ. Press 1990; P. Needham: "Stuff", Australasian Journal of Philosophy, 71 (1993), 270-90; D.W. Zimmerman: "Theories of Masses and Problems of Constitution”, The Philosophical Review, 104 (1995), 53-110; M.C. Rea: "The Problem of Material Constitution", The Philosophical Review, 104 (1995), 525-52.

[2] Historically instructive is E. McMullin: "The Concept of Matter in Transition", in: The Concept of Matter in Modern Philosophy, Notre Dame-London: Univ. of Notre Dame Press 1978, pp. 1-55.

[3] For more details cf. J. Schummer: "Physical Chemistry - Neither Fish nor Fowl?”, in: P. Janich, N. Psarros (eds.), The Autonomy of Chemistry, Wuerzburg: Koenigshausen \& Neumann, 1998.

[4] J. Schummer: "Towards a Philosophy of Chemistry", Journal for General Philosophy of Science, 28 (1997), 307-35.

[5] J. Schummer: "Scientometric Studies on Chemistry I: The Exponential Growth of Chemical Substances”, Scientometrics, 39 (1997), 107-23; and "Challenging Standard Distinctions between Science and Technology: The Case of Preparative Chemistry", HYLE - An 
International Journal for the Philosophy of Chemistry, 3 (1997), 8194.

[6] J. Schummer, Realismus und Chemie. Philosophische Untersuchungen der Wissenschaft von den Stoffen, Wuerzburg: Koenigshausen \& Neumann 1996, sect. 6.6.

[7] J. Schummer: "Die stoffliche Weltveraenderung der Chemie: Philosophische Herausforderungen", in: Proceedings of the 17th German Congress of Philosophy, Leipzig 1996, vol. 1, 429-36.

[8] H. Putnam: "The Meaning of 'Meaning'", in his: Mind, Language and Reality, Cambridge: Cambridge Univ. Press, 1975.

[9] K. Marx, F. Engels, Werke, Berlin 1996, vol. 21, p. 276.

[10] I. Hacking, Representing and Intervening, Cambridge: Cambridge Univ. Press, 1983. 